\title{
Effect of Organosulfur Compounds from Different Garlic Preparations on Hyperlipidemia: An in-silico Approach
}

\author{
Bahman Nickavar ${ }^{1, * \text { (D) }}$ \\ 1 Department of Pharmacognosy, School of Pharmacy, Shahid Beheshti University of Medical Sciences; \\ bnickavar@sbmu.ac.ir (B.N.); \\ * Correspondence: bnickavar@sbmu.ac.ir (B.N.);
}

Scopus Author ID 6602601319

Received: 13.06.2021; Revised: 16.07.2021; Accepted: 22.07.2021; Published: 13.08.2021

\begin{abstract}
Garlic is one of the most well-known medicinal plants in terms of hypolipidemic activities. Its organosulfur compounds (OSCs) are probably one of the major groups of potentially active compounds for treating hyperlipidemia. However, the hypolipidemic activities of many garlic OCSs have not been investigated. This study was conducted to predict the potential of garlic OSCs to interact with hyperlipidemia targets and to evaluate their pharmacokinetic properties. 25 OSCs were selected to interact with four major targets of hyperlipidemia named pancreatic lipase, HMG-CoA reductase, PPAR- $\alpha$, and NPC1L1 using the AutoDock Vina program. The pharmacokinetic properties of OSCs were also evaluated by the SwissADME website. The best binding affinity values were obtained for $\gamma$ glutamyl-S-alk(en)ylcysteines. Other OSCs, such as $S$-alk(en)ylcysteine sulfoxides and ajoenes, presented a moderate to high affinity for the studied targets. However, in-silico evaluation of pharmacokinetic properties showed that $\gamma$-glutamyl-S-alk(en)ylcysteines have low absorption. The results displayed that $\gamma$-glutamyl-S-alk(en)ylcysteines can potentially be considered antihyperlipidemic agents mainly influencing the intestinal lumen. Therefore, it seems that fresh whole garlic, thermal dehydrated garlic, and freeze-dried garlic products are probably more potent antihyperlipidemic agents due to their higher levels of $\gamma$-glutamyl-S-alk(en)ylcysteines.
\end{abstract}

Keywords: Allium sativum; antihyperlipidemic activities; garlic; organosulfur compounds; virtual study.

(C) 2021 by the authors. This article is an open-access article distributed under the terms and conditions of the Creative Commons Attribution (CC BY) license (https://creativecommons.org/licenses/by/4.0/).

\section{Introduction}

Hyperlipidemia, defined as an increase in total cholesterol, low-density lipoprotein cholesterol (LDL-C), and/or triglyceride in circulating levels, is the most common form of dyslipidemia [1]. It is one of the key contributing factors to atherosclerosis development, leading to cardiovascular diseases (CVDs) [2].

Hyperlipidemia is a prevalent problem in many countries due to changes occurred in individuals' lifestyles. Therefore, proper nutrition, exercise, smoking cessation, and maintaining a healthy weight are important factors in managing hyperlipidemia [3]. However, if these modifications do not improve lipid profile, medications should be prescribed as a part of the treatment plan. Lipid-lowering agents encompass several drugs, including HMG-CoA reductase inhibitors or statins (e.g., atorvastatin, fluvastatin, etc.), bile acid sequestrants (e.g., colestipol, cholestyramine, etc.), Niemann-Pick C1-like 1 (NPC1L1) inhibitors, or cholesterol absorption inhibitors (e.g., ezetimibe), peroxisome proliferator-activated receptor-alpha (PPAR- $\alpha$ ) activators or fibrates (e.g., fenofibrate, gemfibrozil, etc.), proprotein convertase 
subtilisin/kexin type 9 (PCSK9) inhibitors (e.g., alirocumab, evolocumab, etc.), pancreatic lipase inhibitors (e.g., orlistat), and niacin [4,5]. Although these agents effectively treat hyperlipidemia, regular consumption of these drugs for a long time may result in health problems [5]. Therefore, there is an increasing tendency to search for new medications with minimal side effects, especially natural sources $[6,7]$.

Table 1. Major OSCs in common garlic preparations.

\begin{tabular}{l|l} 
Garlic product & Main organosulfur compounds in the product \\
\hline $\begin{array}{l}\text { Fresh whole garlic, Thermal dehydrated garlic, and Freeze- } \\
\text { dried garlic }\end{array}$ & $\begin{array}{l}\text { Mainly containing: S-Allylcysteine sulfoxide (Alliin) (01), } \gamma \text { - } \\
\text { Glutamyl-S-trans-1-propenylcysteine (02), } \gamma \text {-Glutamyl-S- } \\
\text { allylcysteine (03), S-trans-1-Propenylcysteine sulfoxide (Isoalliin) } \\
(04), \text { S-Methylcysteine sulfoxide (Mettiin) (05), } \gamma \text {-Glutamyl-S- } \\
\text { methylcysteine (06) }\end{array}$ \\
\hline Crushed or cut garlic & $\begin{array}{l}\text { Mainly containing: Diallyl thiosulfinate (Allicin) (07), Methyl } \\
\text { allyl thiosulfinate (08), 1-Propenyl allyl thiosulfinate (09) } \\
\text { and to some extent containing: } \gamma \text {-Glutamyl-S-trans-1- } \\
\text { propenylcysteine (02), } \gamma \text {-Glutamyl-S-allylcysteine (GSAC) (03), } \\
\gamma \text {-Glutamyl-S-methylcysteine (06) }\end{array}$ \\
\hline $\begin{array}{l}\text { Aged garlic extract (Extraction of garlic in aqueous } \\
\text { solutions for several months) }\end{array}$ & $\begin{array}{l}\text { Mainly containing: S-trans-1-Propenylcysteine (010), S- } \\
\text { Allylcysteine (SAC) (011), S-Allylmercaptocysteine (012), S- } \\
\text { Methylcysteine (025) }\end{array}$ \\
\hline $\begin{array}{l}\text { Steam-distilled garlic oil (Steam distillation of crushed } \\
\text { garlic then dissolving the extracted oil in vegetable oil) }\end{array}$ & $\begin{array}{l}\text { Mainly containing: sulfide compounds such as Diallyl disulfide } \\
(013), \text { Diallyl trisulfide (014), Allyl methyl trisulfide (015), Allyl } \\
\text { methyl disulfide (016), Diallyl tetrasulfide (017), Allyl methyl }\end{array}$ \\
& $\begin{array}{l}\text { tetrasulfide (018), Dimethyl trisulfide (019), Diallyl sulfide (020), } \\
\text { etc }\end{array}$ \\
\hline $\begin{array}{l}\text { Oil-extracted garlic macerate (Extraction of homogenized } \\
\text { garlic in vegetable oil) }\end{array}$ & $\begin{array}{l}\text { Mainly containing:E-Ajoene (021), Z-Ajoene (022), 2-Vinyl-4-H- } \\
1,3 \text {-dithiin (023), 3-Vinyl-4-H-1,2-dithiin (024) }\end{array}$
\end{tabular}

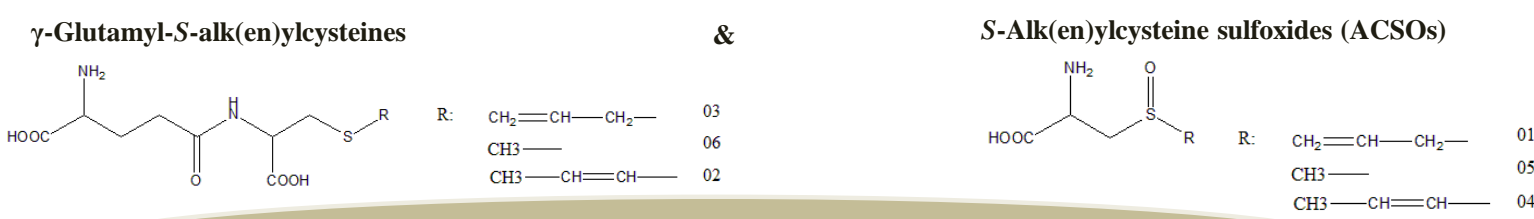

In fresh whole garlic, thermal dehydrated garlic, and freeze-dried garlic

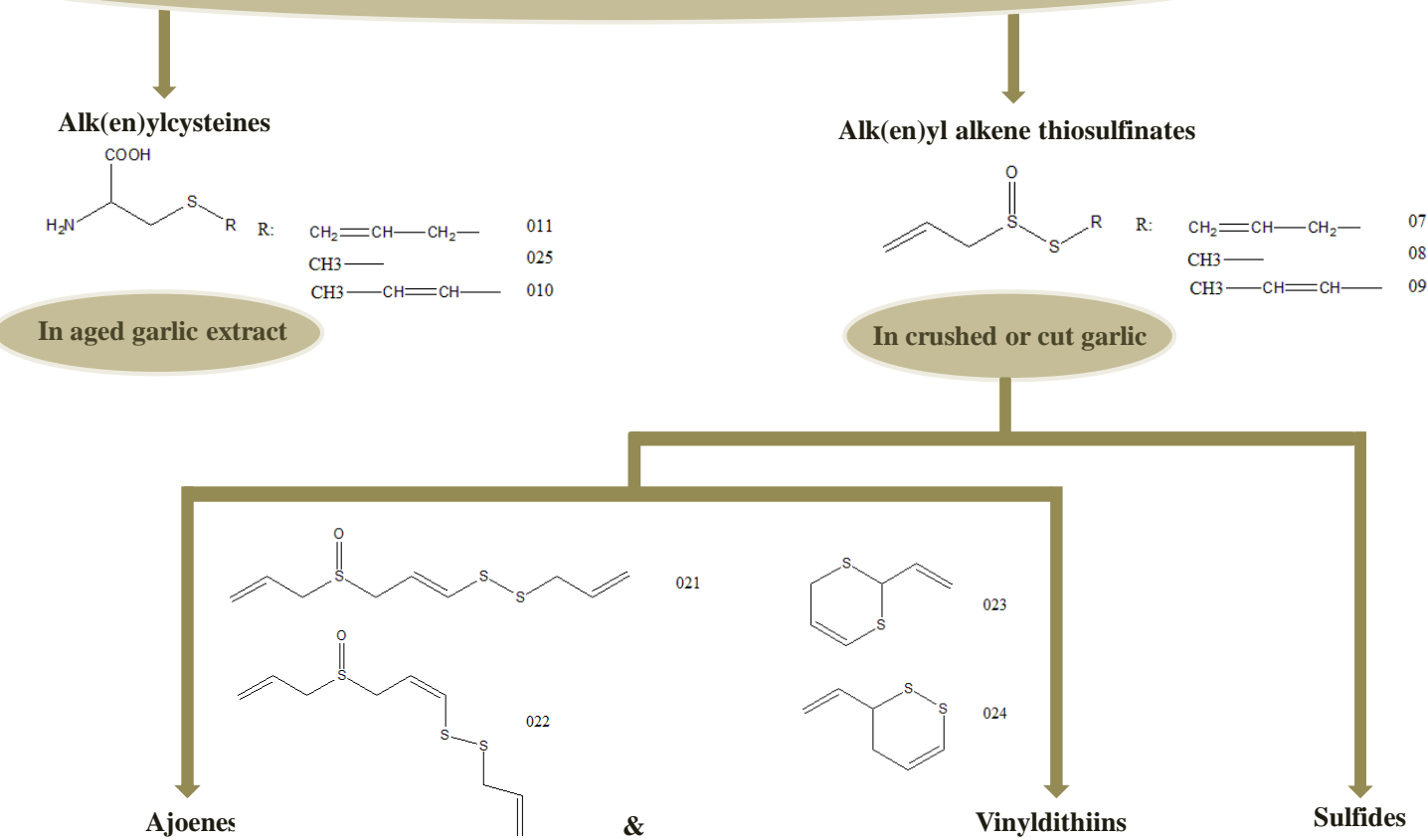

In oil-extracted garlic macerate

In steam-distilled garlic oil

Figure 1. Interconversions among OSCs during the production processes of different garlic products. 
Various studies have shown that the use of medicinal plants has beneficial effects on CVDs [6,7]. In this regard, one of the most well-known plants is garlic [8-10]. Garlic (Allium sativum L.), a member of the Liliaceae family, has long been used for culinary and medical purposes [11]. Different studies have proposed that the health benefits from garlic are largely due to its rich amount of secondary metabolites, such as organosulfur compounds (OSCs), phenolic compounds, saponins, and so on $[12,13]$. OSCs are considered to be responsible for many biological activities and therapeutic potentials of garlic [14]. Various factors such as genotype, growing conditions, irrigation, storage conditions, manufacturing processes, etc., characterize the composition and quantity of OSCs, specifying odor, flavor, and biological activities in garlic $[13,15,16]$. Different studies have indicated that many interconversions can occur among various forms of OSCs during the processing steps of garlic and the production of its preparations (Table 1 and Figure 1). Therefore, various garlic products may differ in biological activities and potential health benefits $[13,17,18]$.

Among the favorable effects of garlic, cardiovascular protection is one of the main functions of garlic-derived products. Several studies have shown that OSCs in garlic plays a fundamental role in reducing the risk of CVDs. OSCs can effectively prevent or improve CVDs due to various pharmacological activities, including antihyperlipidemic, antiatherosclerotic, antihypertension, antiplatelet aggregation, and antithrombotic activities [11,12,14,19-23].

The investigations demonstrate that, in general, garlic can ameliorate hyperlipidemia by regulating serum cholesterol and triglyceride. However, it should be borne in mind that the studies on the influence of garlic on plasma lipid levels have been conducted using various garlic products. Therefore, the type and amount of OSCs have been completely different in these preparations, and as a result, different responses have been given to these products [2426]. Secondly, there are only a limited number of studies on the antihyperlipidemic activities of garlic OSCs. These studies have suggested that hypolipidemic activities of the abovementioned OSCs may be mainly exerted through the reduction of lipid biosynthesis [12,20].

However, as mentioned, the hypolipidemic activities of many OSCs in garlic have not been studied in any way. Therefore, this virtual investigation was carried out to study the influence of the major OSCs in various garlic products against the main therapeutic targets of hyperlipidemia, namely pancreatic lipase, HMG-CoA reductase, PPAR- $\alpha$, and NPC1L1, through molecular docking and evaluating their pharmacokinetic properties.

\section{Materials and Methods}

\subsection{In-Silico molecular docking.}

\subsubsection{Target preparation.}

$\mathrm{X}$-ray crystallographic structures of the protein targets including HMG CoA reductase (PDB ID: 1hwi), PPAR- $\alpha$ (PDB ID: 4bcr), NPC1L1 (PDB ID: 3qnt), and pancreatic lipase (PDB ID: 1lpb) were retrieved from the Protein Data Bank (https://www.rcsb.org/) in PDB format (Table 2). PDB files of targets were simplified by discovery studio visualizer (DSV) software (v.19.1.0.18287). Then, they were prepared for docking study using MGL Tools software (v.1.5.6) and were saved in PDBQT format. Crystallography ligands were used as the reference for the calculation of docking box coordinates [27,28]. 


\begin{tabular}{|c|c|c|c|c|c|c|c|c|}
\hline \multirow[t]{2}{*}{ Target } & \multirow{2}{*}{$\begin{array}{l}\text { PDB } \\
\text { ID } \\
\end{array}$} & \multirow{2}{*}{$\begin{array}{l}\text { Resolution } \\
\left(\mathrm{A}^{\circ}\right)\end{array}$} & \multirow[t]{2}{*}{ Organism } & \multirow{2}{*}{$\begin{array}{l}\text { co-crystallized } \\
\text { ligand }\end{array}$} & \multirow{2}{*}{$\begin{array}{l}\text { Selected } \\
\text { chain }\end{array}$} & \multicolumn{2}{|c|}{ Binding site } & \multirow[t]{2}{*}{ RMSD } \\
\hline & & & & & & Center & Size (A) & \\
\hline $\begin{array}{l}\text { HMG-CoA } \\
\text { reductase }\end{array}$ & 1HWI & 2.30 & Homo sapiens & Fluvastatin & $\mathrm{C}$ & $\begin{array}{l}X=16.9391 \\
Y=16.9294 \\
Z=25.8480\end{array}$ & $\begin{array}{l}X=20 \\
Y=20 \\
Z=20\end{array}$ & 1.607 \\
\hline Pancreatic lipase & 1LPB & 2.46 & Homo sapiens & $\begin{array}{l}\text { Methoxy undecyl } \\
\text { phosphonic acid }\end{array}$ & B & $\begin{array}{l}X=8.8594 \\
Y=23.3771 \\
Z=53.6028\end{array}$ & $\begin{array}{l}X=20 \\
Y=20 \\
Z=20\end{array}$ & 1.876 \\
\hline $\begin{array}{l}\text { Niemann-Pick C1- } \\
\text { like protein } 1 \\
(\mathrm{NPC} 1 \mathrm{~L} 1)\end{array}$ & 3QNT & 2.83 & Homo sapiens & - & $\mathrm{A}$ & $\begin{array}{l}X=-16.3993 \\
Y=-30.6584 \\
Z=-15.6522\end{array}$ & $\begin{array}{l}X=30 \\
Y=30 \\
Z=30\end{array}$ & - \\
\hline $\begin{array}{l}\text { Peroxisome } \\
\text { proliferator- } \\
\text { activated receptor } \\
\text { alpha (PPAR- } \alpha \text { ) }\end{array}$ & 4BCR & 2.50 & Homo sapiens & $\begin{array}{l}\text { Pirinixic acid } \\
\text { (WY14643) }\end{array}$ & A & $\begin{array}{l}X=-20.9449 \\
Y=4.1347 \\
Z=-4.6550\end{array}$ & $\begin{array}{l}X=26 \\
Y=26 \\
Z=26\end{array}$ & 1.943 \\
\hline
\end{tabular}

2.1.2. Ligand preparation.

A list of 25 OSCs was prepared through a literature survey. The list was composed of OSCs reported in various garlic products (Table 1) [14,21,22,29-33]. The 3D structures of OSCs (and other necessary ligands) were retrieved from the PubChem database (https://pubchem.ncbi.nlm.nih.gov) in SDF format and were loaded into PyRx software (v.0.8). PyRx software was used for energy minimization of compounds and converted them into PDBQT format [34].

\subsubsection{Analysis of active sites.}

Ligands bound at active targets in PDB crystal structures can generally help identify and analyze binding positions. For this purpose, identification of active sites on targets (except for NPC1L1) and determination of their properties (including size and location of binding sites) were carried out by DSV software (Table 2) [27]. However, no X-ray crystallographic structure has been previously reported for Homo sapiens NPC1L1 complexed with a ligand (an inhibitor). Therefore, the active site prediction was performed using Molegro Virtual Docker software (v.2013.6.0.1) [35].

\subsubsection{Docking process.}

Initially, docking protocol was validated for each target and its crystallographic ligand by the redocking process. In cases where root mean square deviation (RMSD) was less than 2 (Table 2), the docking process was performed using AutoDock Vina software (v.1.1.2) [36]. For simplification, default settings were implemented in validation and screening simulation. After docking, conformations were analyzed for binding affinity [28]. The modes of interactions between ligands and targets were studied and visualized using DSV software [27]. Orlistate, atorvastatin, fenofibrate, and ezetimibe were used as controls to compare their interactions with the screened OSCs.

\subsection{In-silico pharmacokinetic study.}

Chemical structures of OSCs in Canonical SMILES format were downloaded from PubChem database. Then, they were inserted as input in the SwissADME website to compute 
and anticipate their pharmacokinetics profiles [including absorption (A), distribution (D), metabolism (M), and excretion (E) or ADME] [37].

\section{Results}

\subsection{Antihyperlipidemic activities of garlic-derived OSCs.}

In this investigation, hypolipidemic activities of 25 major OSCs identified in various garlic products were virtually studied against 4 hyperlipidemia targets. All the OSCs exhibited negative binding energy, meaning that they have an affinity for protein targets. However, this affinity was variable with each target. Binding energy range of the OSCs with pancreatic lipase, HMG-CoA reductase, PPAR- $\alpha$, and NPC1L1 was from -2.5 to $-6.2 \mathrm{Kcal} / \mathrm{mol},-2.2--5.1$ $\mathrm{Kcal} / \mathrm{mol},-2.3--5.7 \mathrm{Kcal} / \mathrm{mol}$, and $-2.5--6.5 \mathrm{Kcal} / \mathrm{mol}$, respectively (Table 3 ). In addition, a comparison of the interactions between the top three OSCs-targets in poses with ligands-targets interactions in PDB crystal structures showed that OSCs bind at the desired binding pockets. However, the number and type of interactions should be considered to influence the binding affinity of ligands for targets.

Table 3. Binding energy values of the OSCs against therapeutic targets of hyperlipidemia.

\begin{tabular}{|c|c|c|c|c|c|}
\hline \multirow[t]{2}{*}{ Ligand no. } & \multirow[t]{2}{*}{ Name } & \multicolumn{4}{|c|}{ Binding energy (Kcal/mol) } \\
\hline & & $\begin{array}{l}\text { Pancreatic } \\
\text { lipase }\end{array}$ & $\begin{array}{l}\text { HMG CoA } \\
\text { reductase }\end{array}$ & PPAR- $\alpha$ & NPC1L1 \\
\hline 01 & $S$-Allylcysteine sulfoxide (Alliin) & -4.9 & -4.3 & -4.8 & -5.4 \\
\hline 02 & $\gamma$-Glutamyl-S-trans-1-propenylcysteine & -6.1 & -4.9 & -5.5 & -6.5 \\
\hline 03 & $\gamma$-Glutamyl-S-allylcysteine & -6.2 & -5.1 & -5.7 & -6.2 \\
\hline 04 & $\begin{array}{l}\text { S-trans-1-Propenylcysteine sulfoxide } \\
\text { (Isoalliin) }\end{array}$ & -5.1 & -4.5 & -4.9 & -5.7 \\
\hline 05 & S-Methylcysteine sulfoxide (Mettiin) & -4.6 & -3.8 & -4.1 & -4.8 \\
\hline 06 & $\gamma$-Glutamyl-S-methylcysteine & -6.2 & -4.8 & -5.3 & -6.2 \\
\hline 07 & Diallyl thiosulfinate (Allicin) & -4.3 & -3.4 & -4.4 & -4.6 \\
\hline 08 & Methyl allyl thiosulfinate & -3.6 & -3.1 & -3.9 & -4.0 \\
\hline 09 & 1-Propenyl allyl thiosulfinate & -3.6 & -3.6 & -4.6 & -4.6 \\
\hline 010 & S-trans-1-Propenylcysteine & -5.0 & -4.4 & -4.5 & -5.1 \\
\hline 011 & S-Allylcysteine & -4.7 & -4.3 & -4.4 & -5.0 \\
\hline 012 & S-Allylmercaptocysteine & -4.9 & -3.9 & -4.5 & -5.1 \\
\hline 013 & Diallyl disulfide & -3.8 & -3.2 & -3.9 & -3.9 \\
\hline 014 & Diallyl trisulfide & -4.0 & -3.1 & -4.0 & -4.1 \\
\hline 015 & Allyl methyl trisulfide & -3.5 & -2.8 & -3.5 & -3.5 \\
\hline 016 & Allyl methyl disulfide & -3.2 & -2.6 & -3.3 & -3.3 \\
\hline 017 & Diallyl tetrasulfide & -4.1 & -3.0 & -4.0 & -4.1 \\
\hline 018 & Allyl methyl tetrasulfide & -3.7 & -3.0 & -3.7 & -3.6 \\
\hline 019 & Dimethyl trisulfide & -2.5 & -2.2 & -2.3 & -2.6 \\
\hline 020 & Diallyl sulfide & -3.8 & -3.0 & -3.7 & -3.6 \\
\hline 021 & $E$-Ajoene & -5.1 & -3.4 & -4.9 & -5.2 \\
\hline 022 & Z-Ajoene & -5.0 & -3.5 & -4.9 & -5.2 \\
\hline 023 & 2-Vinyl-4-H-1,3-dithiin & -4.2 & -3.4 & -4.2 & -4.4 \\
\hline 024 & 3-Vinyl-4-H-1,2-dithiin & -4.2 & -3.5 & -4.5 & -4.5 \\
\hline 025 & $S$-Methylcysteine & -4.3 & -3.4 & -3.9 & -4.4 \\
\hline
\end{tabular}

Among 25 studied OSCs, $\gamma$-glutamyl-S-allylcysteine $(-5.1 \mathrm{Kcal} / \mathrm{mol}), \gamma$-glutamyl-Strans-1-propenylcysteine $(-4.9 \mathrm{Kcal} / \mathrm{mol})$, and $\gamma$-glutamyl-S-methylcysteine $(-4.8 \mathrm{Kcal} / \mathrm{mol})$ demonstrated a stronger binding affinity for the HMG-CoA reductase. They could create a network of molecular interactions with various residues at the active site (Table 4 and Figure 2). 
Table 4. Main molecular interactions of top three OSCs with HMG-CoA reductase.

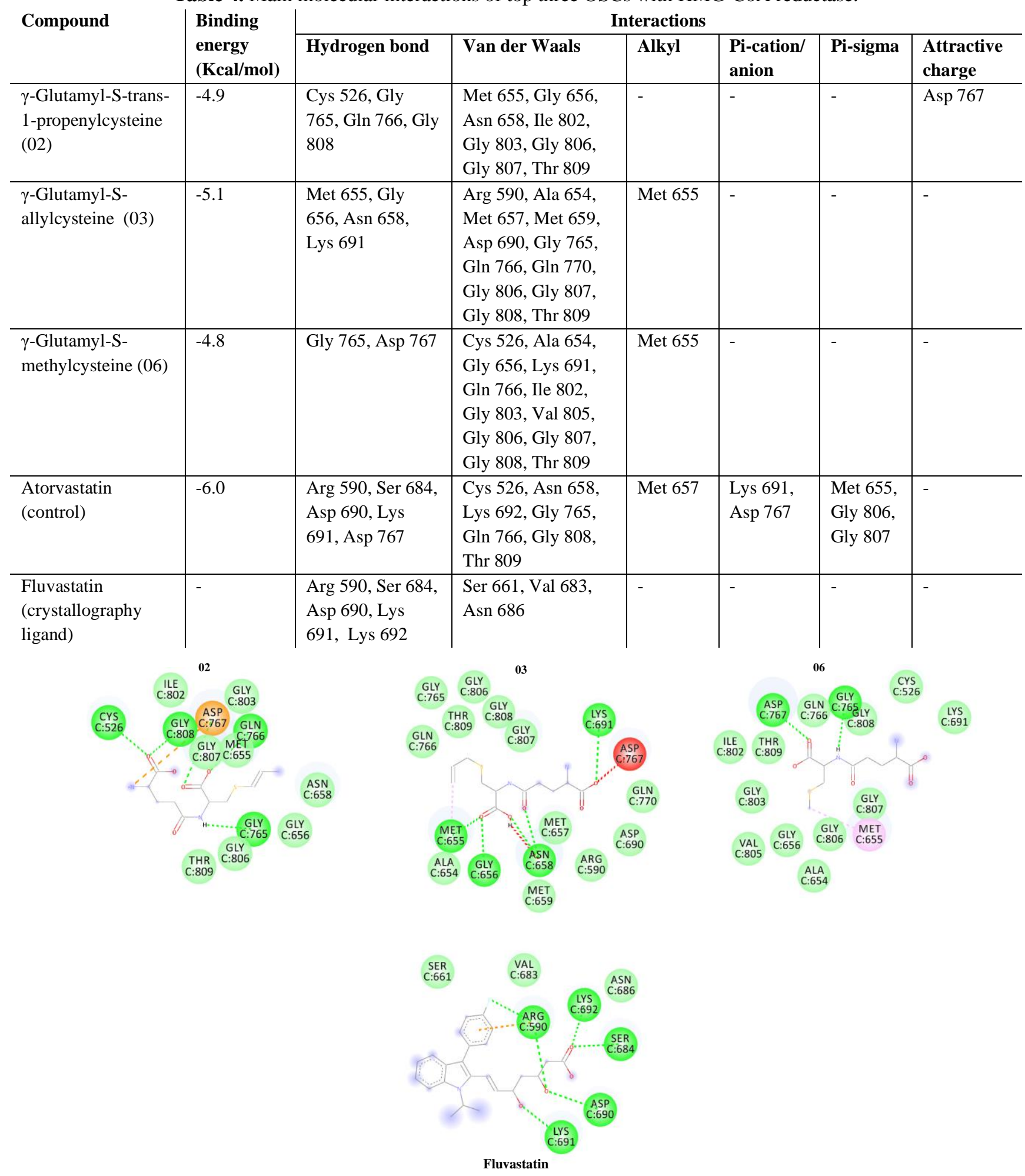

Figure 2. 2D interactions of top three OSCs against HMG-CoA reductase.

The docking results for OSCs tested against pancreatic lipase exhibited that $\gamma$ Glutamyl-S-allylcysteine $(-6.2 \mathrm{Kcal} / \mathrm{mol}), \gamma$-glutamyl-S-methylcysteine $(-6.2 \mathrm{Kcal} / \mathrm{mol})$, and $\gamma$-glutamyl-S-trans-1-propenylcysteine $(-6.1 \mathrm{Kcal} / \mathrm{mol})$ bind more efficiently to the enzyme. They could establish different types of interactions with several residues at the catalytic site (Table 5 and Figure 3). 
Table 5. Main molecular interactions of top three OSCs with pancreatic lipase.

\begin{tabular}{|c|c|c|c|c|c|c|}
\hline \multirow[t]{2}{*}{ Compound } & \multirow{2}{*}{$\begin{array}{l}\text { Binding } \\
\text { energy } \\
(\text { Kcal/mol })\end{array}$} & \multicolumn{5}{|c|}{ Interactions } \\
\hline & & $\begin{array}{l}\text { Covalent } \\
\text { bond }\end{array}$ & Hydrogen bond & Van der Waals & Alkyl & Pi-sigma \\
\hline $\begin{array}{l}\gamma \text {-Glutamyl-S- } \\
\text { trans-1- } \\
\text { propenylcystein } \\
\text { e }(02)\end{array}$ & -6.1 & - & Phe 77, Ser 152 & $\begin{array}{l}\text { His } 75 \text {, Gly } 76, \\
\text { Asp } 79 \text {, Tyr } 114, \\
\text { His } 151 \text {, Leu } 153 \text {, } \\
\text { Ala } 178 \text {, Glu } 179 \text {, } \\
\text { Pro } 180 \text {, Ile } 209 \text {, } \\
\text { Arg } 256 \text {, Ala } 259 \text {, } \\
\text { His } 263 \text {, Leu } 264 \text {, }\end{array}$ & Ile 78 & - \\
\hline $\begin{array}{l}\gamma \text {-Glutamyl-S- } \\
\text { allylcysteine } \\
(03)\end{array}$ & -6.2 & - & $\begin{array}{l}\text { Phe } 77 \text {, His } 151 \text {, } \\
\text { Ser } 152, \text { Phe } 215\end{array}$ & $\begin{array}{l}\text { Gly } 76, \text { Ile } 78, \\
\text { Asp } 79, \text { Tyr } 114, \\
\text { Leu, 153, Ala } \\
\text { 178, Glu } 179, \text { Ile } \\
209, \text { Gly } 216 \text {, His } \\
263\end{array}$ & $\begin{array}{l}\text { Arg 256, Ala } \\
259, \text { Ala 260, } \\
\text { Leu } 264\end{array}$ & - \\
\hline $\begin{array}{l}\gamma \text {-Glutamyl-S- } \\
\text { methylcysteine } \\
(06)\end{array}$ & -6.2 & - & $\begin{array}{l}\text { Phe } 77, \text { Asp } 79, \\
\text { His } 151, \text { Ser } 152 \text {, } \\
\text { Phe } 215\end{array}$ & $\begin{array}{l}\text { His } 75 \text {, Gly } 76, \\
\text { Trp } 85 \text {, Tyr } 114, \\
\text { Leu 153, Ala 178, } \\
\text { Glu 179, Pro } 180, \\
\text { Gly 216, Arg } \\
256 \text {, Ala 260, Leu } \\
264\end{array}$ & Ile 78 & - \\
\hline $\begin{array}{l}\text { Orlistate } \\
\text { (control) }\end{array}$ & -6.8 & - & $\begin{array}{l}\text { Gly } 76 \text {, Phe } 77 \text {, } \\
\text { His } 151 \text {, Ser } 152 \text {, } \\
\text { Leu } 153 \text {, Ala } 260 \text {, } \\
\text { His } 263 \text {, }\end{array}$ & $\begin{array}{l}\text { Asp } 79, \text { Ala } 178, \\
\text { Arg } 256, \text { Ala } 259, \\
\text { Ala } 260, \text { Leu } 264\end{array}$ & $\begin{array}{l}\text { Phe } 77, \text { Ile } \\
78, \text { Tyr 114, } \\
\text { Pro 180, Ile } \\
209\end{array}$ & Tyr 114 \\
\hline $\begin{array}{l}\text { Methoxyundecyl } \\
\text { phosphinic acid } \\
\text { (crystallography } \\
\text { ligand) }\end{array}$ & - & Ser 152 & Phe 77, Leu 153 & $\begin{array}{l}\text { Gly } 76 \text {, Tyr } 114, \\
\text { Ser } 152 \text {, Ala } 178, \\
\text { Glu } 179 \text {, Pro } 180 \text {, } \\
\text { Ile } 209 \text {, Leu } 213 \text {, }\end{array}$ & $\begin{array}{l}\text { His } 151, \text { Phe } \\
215, \text { His } 263\end{array}$ & - \\
\hline
\end{tabular}
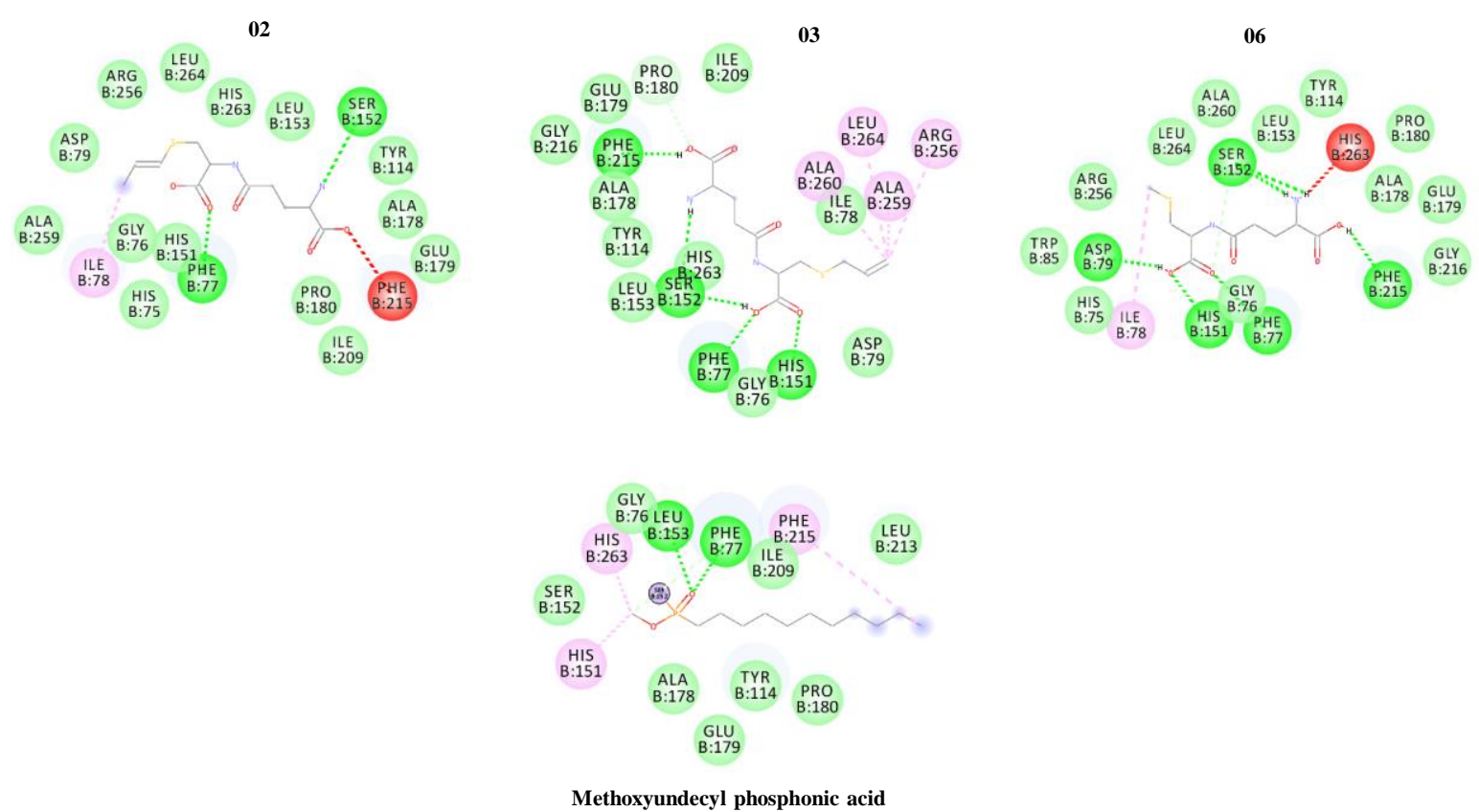

Figure 3. 2D interactions of top three OSCs against pancreatic lipase.

Among 25 studied OSCs, 3 compounds including $\gamma$-glutamyl-S-allylcysteine (-5.7 $\mathrm{Kcal} / \mathrm{mol}), \quad \gamma$-glutamyl-S-trans-1-propenylcysteine $(-5.5 \mathrm{Kcal} / \mathrm{mol})$, and $\gamma$-glutamyl-Smethylcysteine $(-5.3 \mathrm{Kcal} / \mathrm{mol})$ showed the lowest binding energy values with PPAR- $\alpha$. They 
could form several types of interactions with the residues at the binding pocket (Table 6 and Figure 4).

\begin{tabular}{|c|c|c|c|c|c|}
\hline \multirow[t]{2}{*}{ Compound } & \multirow{2}{*}{$\begin{array}{l}\text { Binding } \\
\text { energy } \\
(\text { Kcal/mol }) \\
\end{array}$} & \multicolumn{4}{|c|}{ Interactions } \\
\hline & & $\begin{array}{l}\text { Hydrogen } \\
\text { bond }\end{array}$ & Van der Waals & Alkyl & Pi-sigma \\
\hline $\begin{array}{l}\gamma \text {-Glutamyl-S-trans- } \\
\text { 1-propenylcysteine } \\
(02)\end{array}$ & -5.5 & $\begin{array}{l}\text { Glu 251, Arg } \\
271\end{array}$ & $\begin{array}{l}\text { Ile } 241, \text { Leu } 247 \text {, Ala } 250, \\
\text { Leu } 254, \text { Leu } 258, \text { Ile } 263 \text {, } \\
\text { Lys } 266 \text {, His } 274, \text { Cys } \\
275, \text { Val } 332\end{array}$ & Val 255, Ala 333 & - \\
\hline $\begin{array}{l}\gamma \text {-Glutamyl-S- } \\
\text { allylcysteine }(03)\end{array}$ & -5.7 & $\begin{array}{l}\text { Ser } 280, \text { Thr } \\
283\end{array}$ & $\begin{array}{l}\text { Cys } 275, \text { Gln 277, Thr } \\
\text { 279, Tyr 314, Ile 317, Phe } \\
\text { 318, Leu 321, Val 324, } \\
\text { Leu 331, Met 355, His } \\
\text { 440, Tyr } 464\end{array}$ & Cys 276, Met 330, Val 332, & - \\
\hline $\begin{array}{l}\gamma \text {-Glutamyl-S- } \\
\text { methylcysteine (06) }\end{array}$ & -5.3 & $\begin{array}{l}\text { Ser } 280, \text { Tyr } \\
314, \text { Tyr } 464\end{array}$ & $\begin{array}{l}\text { Cys 276, Gln 277, Thr } \\
\text { 279, Ile 317, Phe 318, Leu } \\
\text { 321, Met 355, His 440, } \\
\text { Leu } 460\end{array}$ & Phe 273, Ile 354, Val 444, & - \\
\hline $\begin{array}{l}\text { Fenofibrate } \\
\text { (control) }\end{array}$ & -7.5 & - & $\begin{array}{l}\text { Glu 251, Leu 254, Glu } \\
282\end{array}$ & $\begin{array}{l}\text { Ile 241, Leu 247, Ala 250, } \\
\text { Ile 272, Cys 275, Cys 278, } \\
\text { Val 332, Ala 333, Tyr } 334 \text {, } \\
\text { Ile } 339\end{array}$ & Val 255 \\
\hline $\begin{array}{l}\text { Pirinixic acid (or } \\
\text { WY14643) } \\
\text { (crystallography } \\
\text { ligand) }\end{array}$ & - & $\begin{array}{l}\text { Glu 251, His } \\
274, \text { Cys } 278\end{array}$ & Leu 254, Val 255, Lys 266 & $\begin{array}{l}\text { Ile 241, Leu 247, Leu 258, } \\
\text { Ile 263, Arg 271, Ile 272, } \\
\text { Cys 275, Val } 332\end{array}$ & - \\
\hline
\end{tabular}
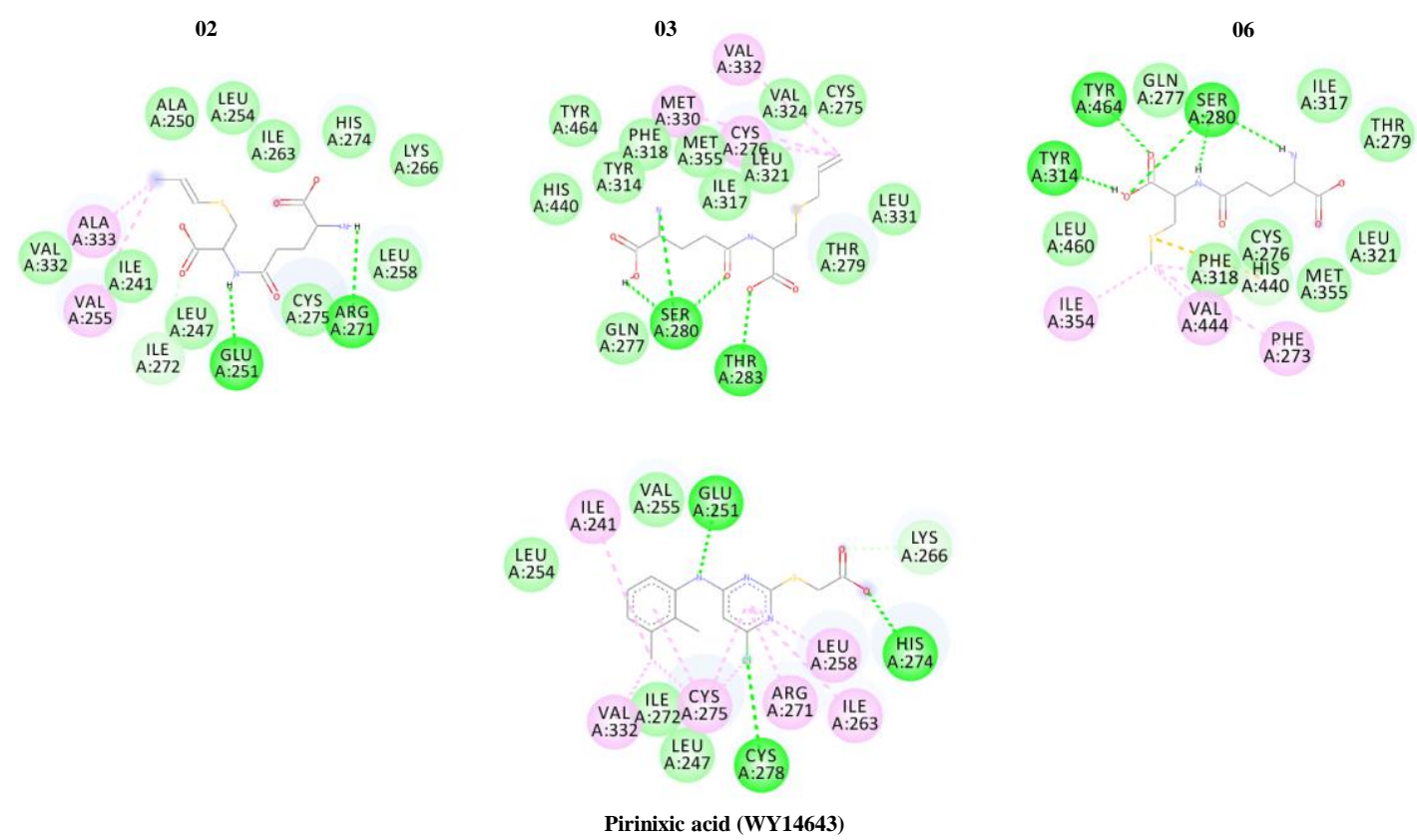

Figure 4. 2D interactions of top three OSCs against PPAR- $\alpha$.

Results of molecular docking analysis of the OSCs studied against NPC1L1 indicated that $\gamma$-glutamyl-S-trans-1-propenylcysteine $(-6.5 \mathrm{Kcal} / \mathrm{mol}), \gamma$-glutamyl-S-allylcysteine $(-6.2$ $\mathrm{Kcal} / \mathrm{mol})$, and $\gamma$-glutamyl-S-methylcysteine $(-6.2 \mathrm{Kcal} / \mathrm{mol})$ have the greatest affinity for the selected target. They could make numerous molecular interactions with the active site residues (Table 7 and Figure 5).

Table 7. Main molecular interactions of top three OSCs with NPC1L1. 


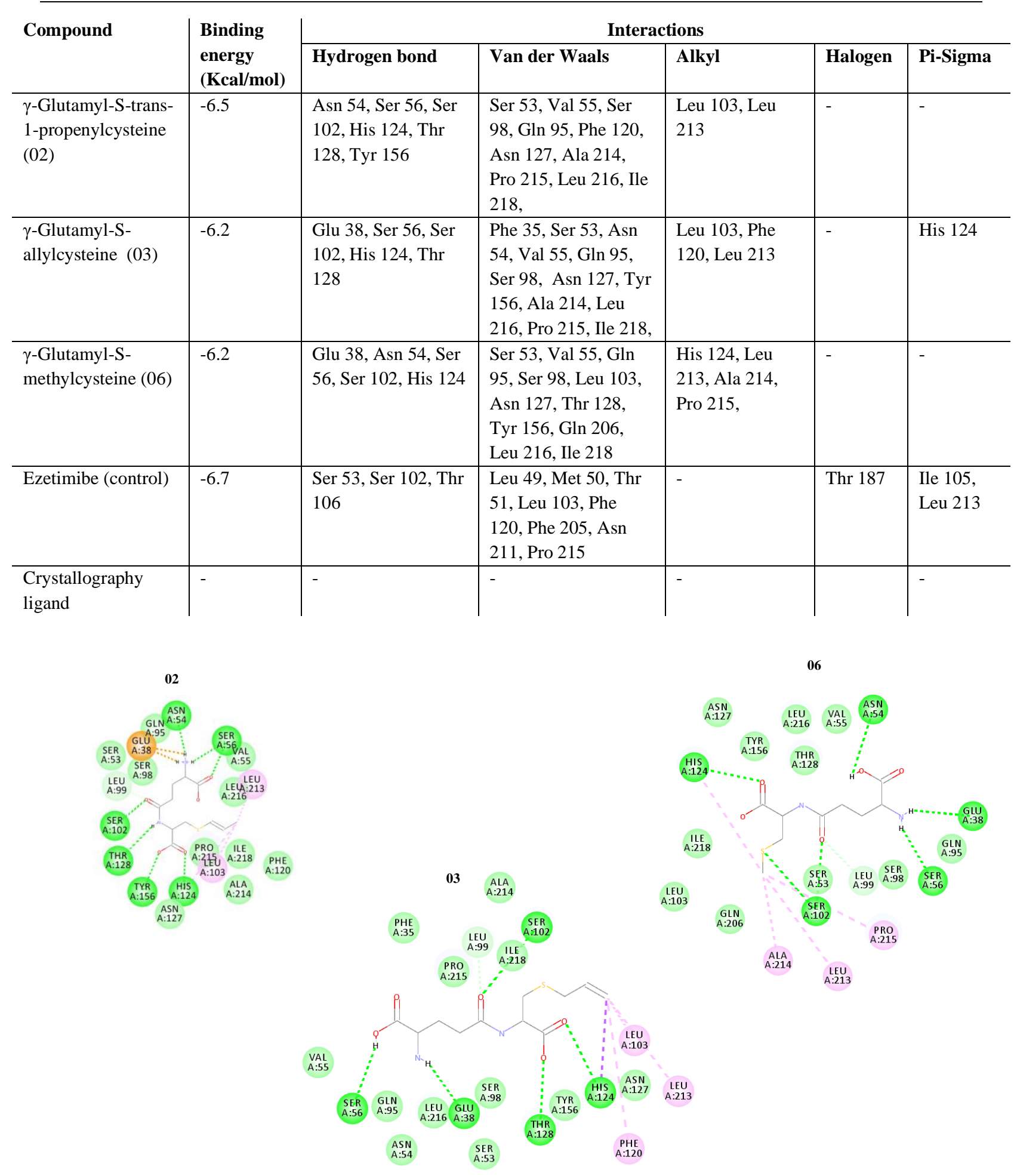

Figure 5. 2D interactions of top three OSCs against NPC1L1.

\subsection{ADME analysis.}

The ADME analysis provides a preliminary assessment of how the body will act in response to a compound. For this purpose, this study was performed on the OSCs investigated in this work, and several properties, such as gastrointestinal (GI) absorption, blood-brain barrier permeation, interaction with P-glycoprotein (P-gp), and inhibition of cytochrome P450 isoforms were predicted. The results of which are presented in Table 8. Based on the presented predictions, most compounds had an acceptable range of ADME properties. 


\begin{tabular}{|c|c|c|c|c|c|c|c|c|c|c|}
\hline $\begin{array}{l}\text { Ligand } \\
\text { no. }\end{array}$ & $\begin{array}{l}\text { Molecular } \\
\text { formula }\end{array}$ & Name & $\begin{array}{l}\text { GI } \\
\text { absorption }\end{array}$ & $\begin{array}{l}\text { BBB } \\
\text { permeation }\end{array}$ & $\begin{array}{l}\text { P-gp } \\
\text { substrate }\end{array}$ & $\begin{array}{l}\text { CYP1A2 } \\
\text { inhibitor }\end{array}$ & $\begin{array}{l}\text { CYP2C19 } \\
\text { inhibitor }\end{array}$ & $\begin{array}{l}\text { CYP2C9 } \\
\text { inhibitor }\end{array}$ & $\begin{array}{l}\text { CYP2D6 } \\
\text { inhibitor }\end{array}$ & $\begin{array}{l}\text { CYP3A4 } \\
\text { inhibitor }\end{array}$ \\
\hline 01 & $\mathrm{C}_{6} \mathrm{H}_{11} \mathrm{NO}_{3} \mathrm{~S}$ & S-Allylcysteine sulfoxide & High & No & No & No & No & No & No & No \\
\hline 02 & $\mathrm{C}_{11} \mathrm{H}_{18} \mathrm{~N}_{2} \mathrm{O}_{5} \mathrm{~S}$ & $\begin{array}{l}\gamma \text {-Glutamyl-S-trans-1- } \\
\text { propenylcysteine }\end{array}$ & Low & No & No & No & No & No & No & No \\
\hline 03 & $\mathrm{C}_{11} \mathrm{H}_{18} \mathrm{~N}_{2} \mathrm{O}_{5} \mathrm{~S}$ & $\gamma$-Glutamyl-S-allylcysteine & Low & No & No & No & No & No & No & No \\
\hline$\overline{04}$ & $\mathrm{C}_{6} \mathrm{H}_{11} \mathrm{NO}_{3} \mathrm{~S}$ & $\begin{array}{l}\text { S-trans-1-Propenylcysteine } \\
\text { sulfoxide }\end{array}$ & High & No & No & No & No & No & No & No \\
\hline 05 & $\mathrm{C}_{4} \mathrm{H}_{9} \mathrm{NO}_{3} \mathrm{~S}$ & $S$-Methylcysteine sulfoxide & High & No & No & No & No & No & No & No \\
\hline 06 & $\mathrm{C}_{9} \mathrm{H}_{16} \mathrm{~N}_{2} \mathrm{O}_{5} \mathrm{~S}$ & $\begin{array}{l}\gamma \text {-Glutamyl-S- } \\
\text { methylcysteine }\end{array}$ & Low & No & No & No & No & No & No & No \\
\hline 07 & $\mathrm{C}_{6} \mathrm{H}_{10} \mathrm{OS}_{2}$ & Diallyl thiosulfinate & High & Yes & No & No & No & No & No & No \\
\hline 08 & $\mathrm{C}_{4} \mathrm{H}_{8} \mathrm{OS}_{2}$ & Methyl allyl thiosulfinate & High & Yes & No & No & No & No & No & No \\
\hline 09 & $\mathrm{C}_{6} \mathrm{H}_{10} \mathrm{OS}_{2}$ & $\begin{array}{l}\text { 1-Propenyl allyl } \\
\text { thiosulfinate }\end{array}$ & High & Yes & No & No & No & No & No & No \\
\hline 010 & $\mathrm{C}_{6} \mathrm{H}_{11} \mathrm{NO}_{2} \mathrm{~S}$ & S-trans-1-Propenylcysteine & High & No & No & No & No & No & No & No \\
\hline 011 & $\mathrm{C}_{6} \mathrm{H}_{11} \mathrm{NO}_{2} \mathrm{~S}$ & $S$-Allylcysteine & High & No & No & No & No & No & No & No \\
\hline 012 & $\mathrm{C}_{6} \mathrm{H}_{11} \mathrm{NO}_{2} \mathrm{~S}_{2}$ & $S$-Allylmercaptocysteine & High & No & No & No & No & No & No & No \\
\hline 013 & $\mathrm{C}_{6} \mathrm{H}_{10} \mathrm{~S}_{2}$ & Diallyl disulfide & High & Yes & No & No & No & No & No & No \\
\hline 014 & $\mathrm{C}_{6} \mathrm{H}_{10} \mathrm{~S}_{3}$ & Diallyl trisulfide & High & Yes & No & No & No & No & No & No \\
\hline 015 & $\mathrm{C}_{4} \mathrm{H}_{8} \mathrm{~S}_{3}$ & Allyl methyl trisulfide & High & Yes & No & No & No & No & No & No \\
\hline 016 & $\mathrm{C}_{4} \mathrm{H}_{8} \mathrm{~S}_{2}$ & Allyl methyl disulfide & High & Yes & No & No & No & No & No & No \\
\hline 017 & $\mathrm{C}_{6} \mathrm{H}_{10} \mathrm{~S}_{4}$ & Diallyl tetrasulfide & High & No & No & No & No & Yes & No & No \\
\hline 018 & $\mathrm{C}_{4} \mathrm{H}_{8} \mathrm{~S}_{4}$ & Allyl methyl tetrasulfide & High & No & No & No & No & Yes & No & No \\
\hline 019 & $\mathrm{C}_{2} \mathrm{H}_{6} \mathrm{~S}_{3}$ & Dimethyl trisulfide & High & Yes & No & No & No & No & No & No \\
\hline 020 & $\mathrm{C}_{6} \mathrm{H}_{10} \mathrm{~S}$ & Diallyl sulfide & High & Yes & $\mathrm{No}$ & $\mathrm{No}$ & No & No & No & No \\
\hline 021 & $\mathrm{C}_{9} \mathrm{H}_{14} \mathrm{OS}_{3}$ & $E$-Ajoene & High & No & $\mathrm{No}$ & $\mathrm{No}$ & $\mathrm{No}$ & Yes & No & No \\
\hline 022 & $\mathrm{C}_{9} \mathrm{H}_{14} \mathrm{OS}_{3}$ & Z-Ajoene & High & No & No & No & No & Yes & No & No \\
\hline 023 & $\mathrm{C}_{6} \mathrm{H}_{8} \mathrm{~S}_{2}$ & 2-Vinyl-4- $H$-1,3-dithiin & High & Yes & No & No & No & No & No & No \\
\hline 024 & $\mathrm{C}_{6} \mathrm{H}_{8} \mathrm{~S}_{2}$ & 3-Vinyl-4- $H$-1,2-dithiin & High & Yes & No & No & No & No & No & No \\
\hline 025 & $\mathrm{C}_{4} \mathrm{H}_{9} \mathrm{NO}_{2} \mathrm{~S}$ & S-Methylcysteine & High & No & No & No & No & No & No & No \\
\hline
\end{tabular}

GI absorption: gastrointestinal absorption; BBB permeation: blood-brain barrier permeation; P-gp substrate: P-glycoprotein substrate. 
However, it was found that only $\gamma$-glutamyl-S-alk(en)ylcysteines (i.e., $\gamma$-glutamyl-Strans-1-propenylcysteine, $\gamma$-glutamyl-S-allylcysteine, and $\gamma$-glutamyl-S-methylcysteine) have low GI absorption. The results also showed that alk(en)yl alkene thiosulfinates [i.e., diallyl thiosulfinate, methyl allyl thiosulfinate, and 1-propenyl allyl thiosulfinate], vinyldithiins, and sulfide compounds (except for tetrasulfide compounds) are not able to cross the blood-brain barrier. On the other hand, it was determined that none of the OSCs acted as substrates for Pglycoprotein and/or as efficient inhibitors for CYP450 isoforms other than ajoenes and tetrasulfide compounds for CYP2C9 isoform.

\section{Discussion}

Based on the above findings, $\gamma$-glutamyl-S-alk(en)ylcysteines have a higher affinity for all targets than the other OSCs. They are more flexible and have more hydrogen-bond acceptor and/or donor sites. Besides, there are $S$-alk(en)ylcysteine sulfoxides and, to some extent, ajoenes. These last two groups exhibited a moderate to a high binding affinity for the targets. However, it should be considered that ajoenes showed a relatively lower affinity for the enzyme HMG-CoA reductase compared to $S$-alk(en)ylcysteine sulfoxides. All the other OSCs displayed a low binding affinity for 4 targets. Furthermore, docking results indicated that 2 out of 4 targets, i.e., pancreatic lipase and NPC1L1 are potentially more susceptible targets to OSCs, especially $\gamma$-glutamyl-S-alk(en)ylcysteines. To the extent of my knowledge, only a few studies have explored the mechanism of the lipid-lowering action of garlic. According to the results of these studies, garlic act mainly by reducing the production of lipids, especially cholesterol [33,38-43]. From a pharmacokinetic point of view, it has been found that the OSCs in garlic are extensively metabolized in the body (after gastrointestinal absorption) and transformed into other sulfur compounds [44-48].

According to the results of the present study, on the one hand, some OSCs from garlic (mainly $\gamma$-glutamyl- $S$-alk(en)ylcysteines and, to a lesser extent $S$-alk(en)ylcysteine sulfoxides and ajoenes) have antihyperlipidemic activities through different mechanisms, especially inhibition of pancreatic lipase and NPC1L1. On the other hand, GI absorption of $\gamma$-glutamyl$S$-alk(en)ylcysteines is relatively low. Then, it can be inferred that a significant part of antihyperlipidemic activities of garlic products may be locally exerted in the intestinal lumen. Also, it seems that the products containing fresh whole garlic, thermal dehydrated garlic, and freeze-dried garlic products may possess more antihyperlipidemic activities due to the higher levels of $\gamma$-glutamyl- $S$-alk(en)ylcysteines and, to some extent, $S$-alk(en)ylcysteine sulfoxides considering the chemical structure of sulfur compounds in different garlic products.

\section{Conclusions}

In this investigation, the OSCs present in different garlic products was virtually studied for antihyperlipidemic activities against various therapeutic targets of hyperlipidemia, including pancreatic lipase, HMG-CoA reductase, PPAR- $\alpha$, and NPC1L1. Initially, 25 OSCs were studied based on their binding affinity for the 4 above-mentioned targets. Then, they were tested for their pharmacokinetic properties. Among the screened OSCs, $\gamma$-glutamyl-Salk(en)ylcysteines displayed the highest binding affinity for all targets, especially pancreatic lipase and NPC1L1. On the contrary, their GI absorption was low, suggesting that, firstly, a substantial part of antihyperlipidemic activities of garlic products are probably exerted directly in the intestinal lumen, and secondly, fresh whole garlic, thermal dehydrated garlic, and freeze- 
dried garlic products may have more antihyperlipidemic activities due to their higher levels of $\gamma$-glutamyl-S-alk(en)ylcysteines.

\section{Funding}

This research received no external funding.

\section{Acknowledgments}

The authors would like to acknowledge the Shahid Beheshti University of Medical Sciences for providing the necessary facilities for this study.

\section{Conflicts of Interest}

The authors declare no conflict of interest.

\section{References}

1. Hedayatnia, M.; Asadi, Z.; Zare-Feyzabadi, R.; Yaghooti-Khorasani, M.; Ghazizadeh, H.; Ghaffarian-Zirak, R.; Nosrati-Tirkani, A.; Mohammadi-Bajgiran, M.; Rohban, M.; Sadabadi, F.; et al. Dyslipidemia and cardiovascular disease risk among the MASHAD study population. Lipids Health Dis. 2020, 19, https://doi.org/10.1186/s12944-020-01204-y.

2. Arya, N.; Kharjul, M.D.; Shishoo, C.J.; Thakare, V.N.; Jain, K.S. Some molecular targets for antihyperlipidemic drug research. Eur. J. Med. Chem. 2014, 85, 535-568, https://doi.org/10.1016/j.ejmech.2014.08.013.

3. Mannu, G.S.; Zaman, M.J.S.; Gupta, A.; Rehman, H.U.; Myint, P.K. Evidence of lifestyle modification in the management of hypercholesterolemia. Curr. Cardiol. Rev. 2013, 9, 2-14, https://doi.org/10.2174/1573403x11309010002.

4. Zodda, D.; Giammona, R.; Schifilliti, S. Treatment strategy for dyslipidemia in cardiovascular disease prevention: Focus on old and new drugs. Pharmacy 2018, 6, https://doi.org/10.3390/pharmacy6010010.

5. Katzung, B.G.; Trevor, A.J. Basic and Clinical Pharmacology, 14th ed.; McGraw-Hill: New York, 2018.

6. El-Tantawy, W.H.; Temraz, A. Natural products for controlling hyperlipidemia: review. Arch. Physiol. Biochem. 2019, 125, 128-135, https://doi.org/10.1080/13813455.2018.1441315.

7. Bahmani, M.; Mirhoseini, M.; Shirzad, H.; Sedighi, M.; Shahinfard, N.; Rafieian-Kopaei, M. A review on promising natural agents effective on hyperlipidemia. J. Evid.- Based Complement. Alternat. 2015, 20, 228238, https://doi.org/10.1177/2156587214568457.

8. Emamat, H.; Tangestani, H.; Totmaj, A.S.; Ghalandari, H.; Nasrollahzadeh, J. The effect of garlic on vascular function: A systematic review of randomized clinical trials. Clin. Nutr. 2020, 39, 3563-3570, https://doi.org/10.1016/j.clnu.2020.02.016.

9. Sun, Y.-E.; Wang, W.; Qin, J. Anti-hyperlipidemia of garlic by reducing the level of total cholesterol and $\begin{array}{lllll}\text { low-density lipoprotein: A meta-analysis. Medicine } & \text { 2018, }\end{array}$ https://doi.org/10.1097/md.0000000000010255.

10. Netzel, M.E. Garlic: Much more than a common spice. Foods 2020, 9, https://doi.org/10.3390/foods9111544.

11. Hosseini, A.; Hosseinzadeh, H. A review on the effects of Allium sativum (Garlic) in metabolic syndrome. J. Endocrinol. Invest. 2015, 38, 1147-1157, https://doi.org/10.1007/s40618-015-0313-8.

12. Shang, A.; Cao, S.Y.; Xu, X.Y.; Gan, R.Y.; Tang, G.Y.; Corke, H.; Mavumengwana, V.; Li, H.B. Bioactive compounds and biological functions of garlic (Allium sativum L.). Foods 2019, 8, https://doi.org/10.3390/foods8070246.

13. Martins, N.; Petropoulos, S.; Ferreira, I.C.F.R. Chemical composition and bioactive compounds of garlic (Allium sativum L.) as affected by pre- and post-harvest conditions: A review. Food Chem. 2016, 211, 41-50, https://doi.org/10.1016/j.foodchem.2016.05.029.

14. Tocmo, R.; Liang, D.; Lin, Y.; Huang, D. Chemical and biochemical mechanisms underlying the cardioprotective roles of dietary organopolysulfides. Front. Nutr. 2015, 2, https://doi.org/10.3389/fnut.2015.00001.

15. Ludlow, R.A.; Pacenza, M.; Chiappetta, A.; Christofides, S.R.; Evans, G.; Graz, M.; Marti, G.; Rogers, H.J.; Müller, C.T. Storage time and temperature affects volatile organic compound profile, alliinase activity and 
postharvest quality of garlic. Postharvest Biology and Technology 2021, 177, https://doi.org/10.1016/j.postharvbio.2021.111533.

16. Abdelrahman, M.; Hirata, S.; Mukae, T.; Yamada, T.; Sawada, Y.; El-Syaed, M.; Yamada, Y.; Sato, M.; Hirai, M.Y.; Shigyo, M. Comprehensive metabolite profiling in genetic resources of garlic (Allium sativum L.) collected from different geographical regions. Molecules 2021, 26, https://doi.org/10.3390/molecules26051415.

17. Quesada, I.; de Paola, M.; Torres-Palazzolo, C.; Camargo, A.; Ferder, L.; Manucha, W.; Castro, C. Effect of garlic's active constituents in inflammation, obesity and cardiovascular disease. Curr. Hypertens. Rep. 2020, 22, https://doi.org/10.1007/s11906-019-1009-9.

18. Sudhakar, K.; Mishra, V.; Hemani, V.; Verma, A.; Jain, A.; Jain, S.; Charyulu, R.N. Reverse pharmacology of phytoconstituents of food and plant in the management of diabetes: Current status and perspectives. Trends in Food Science and Technology 2021, 110, 594-610, https://doi.org/10.1016/j.tifs.2020.10.024.

19. Sobenin, I.A.; Myasoedova, V.A.; Iltchuk, M.I.; Zhang, D.W.; Orekhov, A.N. Therapeutic effects of garlic in cardiovascular atherosclerotic disease. Chin. J. Nat. Med. 2019, 17, 721-728, https://doi.org/10.1016/s1875-5364(19)30088-3.

20. Batiha, G.E.S.; Beshbishy, A.M.; Wasef, L.G.; Elewa, Y.H.A.; Al-Sagan, A.A.; El-Hack, M.E.A.; Taha, A.E.; Abd-Elhakim, Y.M.; Devkota, H.P. Chemical constituents and pharmacological activities of garlic (Allium sativum L.): A review. Nutrients 2020, 12, https://doi.org/10.3390/nu12030872.

21. Charron, C.S.; Milner, J.A.; Novotny, J.A. Garlic. In Encyclopedia of Food and Health, Caballero, B., Finglas, P.M., Toldra, F., Eds.; Elsevier Inc.: Oxford, 2016; 3, 184-190.

22. Zhao, M.M.; Xiong, S.H.; Zhao, G.D.; Yu, H. Organosulfur compounds in food. In Handbook of Dietary Phytochemicals, Xiao, J., Sarker, S.D., Asakawa, Y., Eds.; Springer: Singapore, 2020; 1-21.

23. Gyawali, D.; Vohra, R.; Orme-Johnson, D.; Ramaratnam, S.; Schneider, R.H. A systematic review and metaanalysis of Ayurvedic herbal preparations for hypercholesterolemia. Medicina 2021, 57, https://doi.org/10.3390/medicina57060546.

24. Chan, W.J.J.; McLachlan, A.J.; Luca, E.J.; Harnett, J.E. Garlic (Allium sativum L.) in the management of hypertension and dyslipidemia - A systematic review. J. Herbal Med. 2020, 19, https://doi.org/10.1016/j.hermed.2019.100292.

25. Banerjee, S.K.; Maulik, S.K. Effect of garlic on cardiovascular disorders: A review. Nutr. J. 2002, 1, 1-14, https://doi.org/10.1186/1475-2891-1-1.

26. Schwingshackl, L.; Missbach, B.; Hoffmann, G. An umbrella review of garlic intake and risk of cardiovascular disease. Phytomedicine 2016, 23, 1127-1133, https://doi.org/10.1016/j.phymed.2015.10.015.

27. Noreen; Ali, R.; Badshah, S.L.; Faheem, M.; Abbasi, S.W.; Ullah, R.; Bari, A.; Jamal, S.B.; Mahmood, H.M.; Haider, A.; et al. Identification of potential inhibitors of Zika virus NS5 RNA-dependent RNA polymerase through virtual screening and molecular dynamic simulations. Saudi Pharm. J. 2020, 28, 1580-1591, https://doi.org/10.1016/j.jsps.2020.10.005.

28. Forli, S.; Huey, R.; Pique, M.E.; Sanner, M.F.; Goodsell, D.S.; Olson, A.J. Computational protein-ligand docking and virtual drug screening with the AutoDock suite. Nature Protocols 2016, 11, 905-919, https://doi.org/10.1038/nprot.2016.051.

29. Yamaguchi, Y.; Kumagai, H. Characteristics, biosynthesis, decomposition, metabolism and functions of the garlic odour precursor, S-allyl-L-cysteine sulfoxide. Exp Ther Med 2020, 19, 1528-1535, https://doi.org/10.3892/etm.2019.8385.

30. Jivishov, E.; Keusgen, M. Can Allium chemical chest be a source of anticancer compounds? Phytochem. Rev. 2020, 19, 1503-1523, https://doi.org/10.1007/s11101-020-09712-6.

31. Rose, P.; Whiteman, M.; Moore, P.K.; Yi, Z.Z. Bioactive S-alk(en)yl cysteine sulfoxide metabolites in the genus Allium: The chemistry of potential therapeutic agents. Nat. Prod. Rep. 2005, 22, 351-368, https://doi.org/10.1039/b417639c.

32. Ahmad, P.; Alvi, S.S.; Salman Khan, M. Functioning of organosulfur compounds from garlic (Allium sativum Linn) in targeting risk factor-mediated atherosclerosis: a cross talk between alternative and modern medicine. In Natural Bio-active Compounds, Akhtar, M.S., Swamy, M.K., Sinniah, U.R., Eds.; Springer: Singapor, 2019; $1,561-586$.

33. Omar, S.H. Garlic and cardiovascular diseases. In Natural Products: Phytochemistry, Botany and Metabolism of Alkaloids, Phenolics and Terpenes, Ramawat, K.G., Merillon, J.M., Eds.; Springer: Berlin, 2013; 36613696. 
34. Dallakyan, S.; Olson, A.J. Small-molecule library screening by docking with PyRx. Methods in Molecular Biology 2015, 1263, 243-250, https://doi.org/10.1007/978-1-4939-2269-7_19.

35. Torktaz, I.; Behjati, M.; Arjmand, S. Virtual screening of M3 protein antagonists for finding a model to study the gammaherpesvirus damaged immune system and chemokine related diseases. BioImpacts 2013, 3, 177 183, https://doi.org/10.5681/bi.2013.022.

36. Plewczynski, D.; Łaźniewski, M.; Augustyniak, R.; Ginalski, K. Can we trust docking results? Evaluation of seven commonly used programs on PDBbind database. J. Comput. Chem. 2011, 32, 742-755, https://doi.org/10.1002/jcc. 21643.

37. Daina, A.; Michielin, O.; Zoete, V. SwissADME: A free web tool to evaluate pharmacokinetics, drug-likeness and medicinal chemistry friendliness of small molecules. Sci. Rep. 2017, 7, https://doi.org/10.1038/srep42717.

38. Gebhardt, R.; Beck, H. Differential inhibitory effects of garlic-derived organosulfur compounds on cholesterol biosynthesis in primary rat hepatocyte cultures. Lipids 1996, 31, 1269-1276, https://doi.org/10.1007/bf02587912.

39. Yeh, Y.Y.; Yeh, S.M. Garlic reduces plasma lipids by inhibiting hepatic cholesterol and triacylglycerol synthesis. Lipids 1994, 29, 189-193, https://doi.org/10.1007/bf02536728.

40. Yeh, Y.Y.; Liu, L. Cholesterol-lowering effect of garlic extracts and organosulfur compounds: Human and animal studies. J. Nutr. 2001, 131, 989S-993S, https://doi.org/10.1093/jn/131.3.989s.

41. Gebhardt, R. Multiple inhibitory effects of garlic extracts on cholesterol biosynthesis in hepatocytes. Lipids 1993, 28, 613-619, https://doi.org/10.1007/bf02536055.

42. Liu, L.; Yeh, Y.Y. S-alk(en)yl cysteines of garlic inhibit cholesterol synthesis by deactivating HMG-CoA reductase in cultured rat hepatocytes. J. Nutr. 2002, 132, 1129-1134, https://doi.org/10.1093/jn/132.6.1129.

43. Mahdavi, A.; Bagherniya, M.; Fakheran, O.; Reiner, Ž.; Xu, S.; Sahebkar, A. Medicinal plants and bioactive natural compounds as inhibitors of HMG-CoA reductase: A literature review. BioFactors 2020, 46, 906-926, https://doi.org/10.1002/biof.1684.

44. Yun, H.M.; Ban, J.O.; Park, K.R.; Lee, C.K.; Jeong, H.S.; Han, S.B.; Hong, J.T. Potential therapeutic effects of functionally active compounds isolated from garlic. Pharmacol. Ther. 2014, 142, 183-195, https://doi.org/10.1016/j.pharmthera.2013.12.005.

45. Berginc, K.; Kristl, A. The effect of garlic supplements and phytochemicals on the ADMET properties of drugs. Expert Opin. Drug Metab. Toxicol. 2012, 8, 295-310, https://doi.org/10.1517/17425255.2012.659662.

46. Rahman, M.S. Allicin and other functional active components in garlic: Health benefits and bioavailability. Int. J. Food Prop. 2007, 10, 245-268, https://doi.org/10.1080/10942910601113327.

47. Gao, C.; Jiang, X.; Wang, H.; Zhao, Z.; Wang, W. Drug metabolism and pharmacokinetics of organosulfur compounds from garlic. J. Drug Metab. Toxicol. 2013, 4, https://doi.org/10.4172/2157-7609.1000159.

48. Iciek, M.; Kwiecień, I.; Włodek, L. Biological properties of garlic and garlic-derived organosulfur compounds. Environ. Mol. Mutagen. 2009, 50, 247-265, https://doi.org/10.1002/em.20474. 